\title{
Impact of exercise training program attendance and physical activity participation on six minute walk distance in patients with heart failure
}

Julie A Adsett

Norman R Morris

Alison M Mudge

\author{
BPhty (Hons) ${ }^{\text {a, b,c }}$ \\ PhD, B Appl Sci (Physiotherapy), Dip Ed, BSc c,d,e \\ PhD, FRACP, MBBS (Hons), Grad Cert Health Econ f,g
}

${ }^{\text {a }}$ Heart Support Service, Royal Brisbane and Women's Hospital, Brisbane, Australia

${ }^{\mathrm{b}}$ Physiotherapy Department, Royal Brisbane and Women's Hospital, Brisbane, Australia

${ }^{\mathrm{c}}$ School of Allied Health Sciences, Griffith University, Gold Coast, Australia

${ }^{\mathrm{d}}$ Menzies Health Institute, Queensland

${ }^{\mathrm{e}}$ Metro North Hospital and Health Service, The Prince Charles Hospital. Allied Health Research

Collaborative

${ }_{\mathrm{f}}^{\mathrm{f}}$ Department of Internal Medicine and Aged Care, Royal Brisbane and Women's Hospital, Brisbane, Australia

${ }^{\mathrm{g}}$ University of Queensland Faculty of Medicine, Brisbane, Queensland, Australia

\section{Corresponding author:}

Julie Adsett,

\section{Email: Julie.adsett@health.qld.gov.au}

Postal address: Heart Support Service, Level 3, James Mayne Building,

Royal Brisbane and Women's Hospital, Butterfield St, Herston, Brisbane, Australia 4029

Ph: +61 $0736460286 \quad$ F: +610736460272

Word count $=3320$ 


\section{Acknowledgements}

The authors would like to acknowledge and thank the EJECTION HF study investigators, Diane Bookless for data collection and the patients and staff of the Heart Failure Services at the participating facilities. We also thank Isabel Hoffman who contributed to statistical analysis of the data.

\section{Sources of funding}

This work was supported by the National Health and Medical Research Council (NHMRC Project grant no. 498403). The primary author was also supported by a Heart Foundation of Australia, post graduate scholarship.

Disclosure statement: The authors report no conflicts of interest 


\section{Introduction}

Exercise training is recommended for all people with stable heart failure (HF) (Sagar et al, 2015). Despite well documented benefits, adherence to this intervention by people with HF is poor (Conraads et al, 2012; Piepoli et al, 2015). Structured, center-based exercise training programs remain the cornerstone of current exercise recommendations, however poor attendance and high drop out rates are common, both of which compromize short and long term outcomes (Conraads et al, 2012; Hammill, Curtis, Schulman, and Whellan, 2010; van der Wal et al, 2010). Adherence to physical activity guidelines is poor in people with HF (Klompstra, Jaarsma, and Stromberg, 2015) and failure to meet these guidelines is associated with poorer clinical outcomes (Doukky et al, 2016). Physical activity incorporates structured exercise training as well as all recreational, occupational and functional activities undertaken by an individual. Current guidelines recommend that individuals undertake 150-300 minutes of moderate intensity activity or 75 - 150 minutes of vigorous intensity activity per week (Eckel et al, 2014) or a combination of both.

Surprisingly little is known about the exercise behaviours of people with HF, and in particular, those recently hospitalized. Many exercise training trials have been conducted in stable and select populations and exercise program adherence is often poorly reported (Deka, Pozehl, Williams, and Yates, 2017). Physical activity undertaken outside of health facilities, and change in physical actvity associated with an intervention, are also rarely described. Several studies have previously sought to identify predictors of exercise behaviour in people with HF. Very few studies however, have specifically investigated the impact of program attendance and physical activity participation, and the inter-relationship between these variables, on change in exercise capacity.Understanding these variables in more detail may assist clinicians 
to deliver exercise training programs specifically targeted for best effect, thereby assisting individuals to make meaningful and sustainable change.

The aim of the current study was to describe patterns of exercise training program attendance and physical activity participation in a sample of recently hospitalized patients enrolled in a comprehensive HF disease management program. An additional aim was to the measure the association between program attendance (frequency and duration of attendance) and physical activity participation with change in six minute walk distance (6MWD). We hypothesised that program attendance variables and physical activity participation would be independently associated with improvements in 6MWD at 12 weeks.

\section{Methods}

\section{Study design and participants}

This study is a secondary analysis of the EJECTION-HF trial (ACTRN12608000263392). The design and primary outcomes of the EJECTION-HF trial, as well as detailed inclusion and exclusion criteria, have been reported previously (Mudge et al, 2018; Mudge et al, 2011). In brief, this multi-center, randomized controlled trial evaluated outcomes from a center-based exercise training program, delivered as a component of a comprehensive disease management program for patients recently (within six weeks) hospitalized with HF. Participants were recruited from HF services at three major metropolitan hospitals and two community facilities in south east Queensland, Australia, from 2008-2013. This secondary analysis is confined to participants in the intervention group only, who were randomized to receive the center-based exercise training program. Informed consent was obtained from all participants. The study complied with the Declaration of Helsinki and was approved by the Human Research Ethics 
Committee (HREC) at each participating site, centrally co-ordinated by the Royal Brisbane and Women's Hospital HREC.

Intervention

All participants were enrolled in a multidisciplinary HF disease management program which included weekly group based education sessions for 12 weeks, individual education and counselling, symptom review and medication titration supported by clinic review and telephone follow-up. Additionally, all participants received supervised center-based exercise training and a comprehensive home exercise program, prescribed and progressed by the physiotherapist and/or accredited exercise physiologist at each site. A program rolling model was employed with the aim that participants commence exercise training within six weeks of hospital discharge. The center-based exercise training program consisted of twice weekly sessions conducted in the facility's gymnasium for 12 weeks (with 24 sessions equalling $100 \%$ attendance), followed by weekly maintenance sessions for an additional 12 weeks. Duration of training was selected on the basis that this is common practice for HF exercise training programs in Australia and was similar to previous research trials (Davidson et al, 2010; O'Connor et al, 2009). Analysis in this study was confined to the initial 12 weeks only. Programs were one hour duration and included a range of upper limb and lower limb endurance and resistance exercises in addition to warm up and cool down. Exercises were performed at an intensity of 9-14 on the rating of perceived exertion (RPE, range 6-20) scale and were monitored and progressed regularly to maintain this intensity.

Participants were also encouraged to undertake a home exercise program consisting of at least 30 minutes of moderate intensity exercise per day (RPE 9-14), for most days of the week, aiming for 150 minutes per week (ie at least 5 days/week). This included a walking program as well as individually prescribed endurance and resistance exercises performed in the home 
environment. Exercises in the home exercise program were often included in the center-based program as a means of ensuring correct exercise intensity and technique. An exercise diary was provided to each participant, and was collected and reviewed weekly by the exercise specialist, at which time an updated version with modifications and progressions was provided.

\section{Outcomes}

\section{Change in 6MWD}

The primary outcome of this secondary study was improvement in 6MWD at 12 weeks. Assessments were conducted at baseline and week 12 according to recommended procedure (Holland et al, 2014), and performed by a single research officer, blinded to allocation. The test was repeated at baseline with the best result (greatest distance), used for analysis. A single 6MWD test was performed at the 12 week assessment. For the purposes of analysis and to ensure clinical relevance of results, 6MWD data were dichotomised according to improvement (yes/ no) whereby improvement was defined as i) an increase in 6MWD at follow up by at least 30 metres (Holland et al, 2014) or ii) no decline in 6MWD if baseline distance walked was greater than 500 metres. This latter condition was included to account for the ceiling effect often observed in this test. No improvement was defined as those who did not meet the above criteria.

Exercise training program attendance

Program attendance was recorded and documented at each exercise session by clinical staff at respective facilities. Total number of sessions was collated by project staff at completion of the intervention period. Attendance frequency was defined as the total number of sessions attended during the 12 week period that the individual undertook supervised exercise training. 
Data were dichotomized into "frequent" and "infrequent attendance" whereby frequent was defined as attendance at $\geq 12$ sessions. Attendance duration was defined as the number of weeks between the first attendance and the last attendance (with no reference to any missing appointments in between) during the 12 week period. Duration thus reflected time to drop out. Data were dichotomized into two groups using a duration of six or more weeks being the cut off.

Physical activity participation

Physical activity participation was defined according to all self-reported activity performed outside of the structured exercise program, and included exercise undertaken as part of the prescribed home exercise program. Participants were provided with examples of activities that represented "moderate intensity" and the project officer clarified responses with participants to optimize accuracy. Those who participated in 150 minutes or more of moderate intensity physical activity per week in the two weeks preceding the respective assessment, were classified as being physically active whilst those who reported not achieving this target, were classified as physically inactive.

Participant characteristics

Participant characteristics such as age, ejection fraction, HF phenotype, HF presentation (new versus decompensated HF), co-morbidities and medications, were obtained from medical records and assessment items undertaken as part of the baseline physical assessment (Geriatric Depression Scale, Instrumental Activities of Daily Living scale).

\section{Data analysis}

Participant characteristics, exercise training program attendance frequency and duration, and physical activity participation at baseline and 12 weeks were summarized using standard 
descriptive statistics. Association between exercise variables and improvement in 6MWD at 12 weeks was described using contingency tables and chi-square testing.

All outcomes were assessed for normality. Logistic regression was then performed to examine the independent association of these exercise variables with improvement in 6MWD at 12 weeks. Results were reported using odds ratios and 95\% confidence intervals, with the level of statistical significance set at $\mathrm{p}<0.05$. Analysis was undertaken using an intention to treat approach. To determine if missing data at week 12 was likely to significantly impact upon results, comparison was made between data from those with complete datasets and data from the whole study group $(\mathrm{n}=140)$, using $\mathrm{t}$ tests for baseline mean 6MWD and age, and a Fisher's exact test for categorical variables.

\section{Results}

One hundred and forty participants were assigned to the intervention arm of EJECTION-HF and provided data for this analysis. Figure 1 demonstrates missing data at each time point. Participants with and without missing data were found to have similar characteristics (data not shown). As demonstrated in Table 1, participants were predominantly male and had a mean age of 62 years. The majority of participants had HF with reduced ejection fraction (HFrEF) and multimorbidity and disability were common.

Of the 140 participants in this study, only one person attended all 24 available sessions in the 12 week period. Fifty-seven (41\%) participants attended frequently ( $\geq 12$ sessions) and median attendance was 9 sessions over the 12 weeks. As depicted in Figure 2, non attendance was most common in the second half of the program. At week one, the majority of participants attended at least once with 67 (48\%) attending one session and 33 (24\%) attending twice as per the protocol. At week 12, only half (53\%) of participants attended, with $46(33 \%)$ attending one session and $28(20 \%)$ attending both sessions offered. The median duration of 
attendance (time to drop out) was 11 weeks, with 58 (41\%) participants attending for the full duration of 12 weeks, and $112(80 \%)$ for at least 6 weeks. At baseline, 60/140 (43\%) participants were physically active. At 12 weeks 92/117 were physically active, including 50 who had been physically inactive at baseline.

The association between exercise variables and improvement in 6MWD is shown in Table 2 . Frequent exercise training program attendance and being physically active at 12 weeks, were both important contributors to significant improvement in 6MWD. Physical activity participation at baseline and attendance duration were not significantly associated with improvement in 6MWD and were therefore not included in the subsequent model. Results of the logistic regression model demonstrated that those who were physically active at week 12 were more likely to improve 6MWD than those not physically active at this time (OR 3.8, 95\% CI 1.3-11.5, $\mathrm{p}=0.018$ ). Similarly, frequent attenders were more likely to improve 6MWD compared to poor attenders (OR 2.7, 95\% CI 1.2-5.9, $\mathrm{p}=0.016)$. Those who achieved both targets were 10.3 times more likely to improve 6MWD. There was no interaction effect between these variables.

Figure 3 illustrates the proportion of participants who improved 6MWD at 12 weeks, with respect to physical activity participation and exercise training program attendance. Those individuals who attended frequently and who were physically active at 12 weeks were significantly more likely to improve 6MWD than those physically inactive at follow up $(p=0.009)$.

\section{Discussion}

This secondary analysis of the EJECTION-HF trial was undertaken to describe exercise participation in people recently hospitalized with HF and to identify associations between exercise participation and improvements in 6MWD. In this study only one participant 
attended all 24 exercise sessions and fewer than half of participants attended $50 \%$ of available sessions at the health facility. At 12 week follow up, close to $80 \%$ of HF participants were deemed to be physically active. Both parameters, exercise training program attendance and meeting physical activity guidelines, were strongly associated with improvements in 6MWD.

Very few studies have specifically investigated exercise participation in people recently hospitalised with HF. Attending more than one exercise session per week was not common in our participants, however compares favourably to a similar trial which aimed to enrol participants within ten days of hospital discharge (Houchen-Wolloff, Watt, Bryce, and Singh, 2016). This latter study found an exercise training program undertaken at this very early time frame to be non feasible due to poor participant uptake (Houchen-Wolloff, Watt, Bryce, and Singh, 2016).

Results from studies enrolling symptomatically stable patients suggest that poor program attendance is indeed a common problem (Collins et al, 2004; Deka, Pozehl, Williams, and Yates, 2017; Mandic et al, 2009; O'Connor et al, 2009; Park et al, 2017). In the post discharge phase, specific challenges may pose as barriers to exercise participation, potentially leading individuals to be negatively labelled as "non adherent". This is particularly relevant for the elderly and those with multimorbidity who are often required to balance multiple competing health services at this time, or who may require readmission to hospital as a consequence of worsening symptoms. In order to account for these challenges and periods of symptom instability, HF exercise training programmes are typically longer in duration than traditional cardiac rehabilitation programmes. Results of this pragmatic trial, in which half of participants had multimorbidity or disability, suggest that whilst recruitment to the exercise program within six weeks of hospital discharge was feasible, it may be unrealistic to expect people to attend more than once per week. 
That meeting physical activity guidelines was strongly associated with improvements in 6MWD is a reassuring result for people unable to attend center-based exercise training programs or for those only able to attend infrequently. These results are also supported by Sutherland et al, who similarly demonstrated a significant association between meeting physical activity guidelines and improvements in 6MWD (Sutherland, Harrison, and Doherty, 2018). In our study, those who achieved both targets of frequent program attendance and being physically active, were 10 times more likely to improve walking distance at 12 week follow up. This "dose" response has been demonstrated previously by Keteyian et al, (2012), and is a worthwhile message with which to counsel patients (Keteyian et al, 2012). For those who are able to attend exercise training programs, frequent attendance should be supported, accompanied by encouragement to undertake a home exercise program at recommended levels. This dose response may also explain the finding that programme duration was not signifiucantly associated with improvements in exercise capacity in this study, a result previously reported by Vromen et al (Vromen et al, 2016).

An interesting finding in the current study was change in physical activity participation following the center-based program. This outcome has rarely been reported in HF exercise trials. In our study, less than half of participants were physically active at baseline, however by 12 weeks, almost $80 \%$ reported meeting this target. This exceeds results of other large center-based trials including the HF ACTION trial, in which only one third of participants were reported to achieve 120 minutes of moderate intensity exercise per week at follow up (O'Connor et al, 2009). Conducting the exercise program as a component of a comprehensive multidisciplinary disease management program, rather than a standalone intervention, may explain these findings. In the current study participants were reviewed at each exercise session attendance by HF Service clinicians, an intervention which included education, monitoring of symptoms, medication review and titration, early management of concerns and provision of 
emotional support. Motivational interviewing techniques were used at each session to review and modify the center-based and home exercise programs as well as to support other behaviour changes in collaboration with the patient. Some of these strategies have previously been recommended for enhancing exercise adherence (Conraads et al, 2012; Deka, Pozehl, Williams, and Yates, 2017). It is possible that incorporating the exercise program as part of a comprehensive service, delivered in one location by a consistent team, may have enhanced individuals' perception of benefit, thus explaining these positive findings compared to previous trials. A recent study by Liu et al, similarly recommends this model of delivery (Liu et al, 2018).

The importance of physical activity undertaken outside of the hospital environment cannot be underestimated. In addition to improving exercise capacity (Evangelista, Cacciata, Stromberg and Dracup, 2017; Sato et al, 2012; Sutherland, Harrison, and Doherty, 2018), it has also been identified as an important predictor of clinical outcomes in people with HF (Doukky et al, 2016; Keteyian et al, 2012; Sato et al, 2012; Waring, Gross, Soucier, and ZuWallack, 2017). Conversely, being physically inactive has been shown to be associated with double the risk of both all cause and cardiac mortality compared to those who are physically active (Doukky et al, 2016). Even modest leisure time activity in HF patients has been associated with improved survival (Doukky et al, 2016; Keteyian et al, 2012). This is an important message to convey to patients. Whilst higher training intensities may yield more promising results, for older patients and those with more severe symptoms or pain, exercising at even moderate intensity may be unrealistic (Haedtke et al, 2017). An approach recently proposed for those with chronic lung disease is to place emphasis on substituting sedentary time with light intensity physical activity, rather than a "one size fits all approach" with recommendations based solely on moderate to vigorous intensity exercise (Cavalheri et al, 2016; Furlanetto et al, 2017; Mesquita et al, 2017; Spruit et al, 2015). Providing clear, "real world" and achievable 
recommendations with tangible outcomes may enhance exercise adherence in these patients (Back, Oberg, and Krevers, 2017; Conraads et al, 2012).

There are several limitations to our study. There is currently no consensus regarding best measurement of physical activity and data from our study were dependent upon a non validated method of self-report. Whilst we considered using accelerometers, we were concerned that this may have influenced exercise behaviour, and may have added to research burden for participants in this study. Attendance data and other outcomes may have been influenced by hospital admissions during the study period as well as other unmeasured confounders. Sample size was relatively small and the study was thus underpowered to include multiple variables in the regression analysis. It should be highlighted that important factors such as baseline characteristics, were not included in the analysis and may have influenced change in 6MWD. Findings should therefore be interpreted with caution. Results also potentially pertain to a select population due to the small sample size, however characteristics of participants were similar to those seen clinically in exercise training programs and results therefore have relevance.

This study demonstrated that meeting physical activity guidelines and attending at least one exercise training session per week were both strongly and independently associated with improvements in 6MWD. Whilst the goals of any exercise intervention should be to improve and maintain exercise capacity, equally important is to promote positive and sustainable exercise behaviours that optimize clinical outcomes in the long term (Conraads et al, 2012; Cooper et al, 2015; Doukky et al, 2016). Services should be tailored for best effect, with interventions directed towards assisting individuals to be physically active, acknowledging that the time required to achieve this change may vary among individuals. Flexible models which account for periods of instability should be encouraged, particularly in the post 
hospitalization phase. Limited data is currently available on how age, gender, HF characteristics and other variables may impact upon exercise adherence in patients with HF. Future studies should thus explore this in more detail and characterize potential subgroups for whom a more tailored approach may be appropriate. In so doing, studies should continue to identify strategies which may assist individuals to attend center-based exercise training programs where possible, to improve physical activity participation and to potentially lessen sedentary behaviour. The impact of illness and rehospitalisation on exercise participation following discharge should also be explored. Finally, studies should explore safety and efficacy of alternative options for exercise training as a means of providing further opportunities for individuals to engage in sustainable and meaningful activity. 


\section{References}

1. Back M, Oberg B, Krevers B 2017 Important aspects in relation to patients' attendance at exercise-based cardiac rehabilitation - facilitators, barriers and physiotherapist's role: a qualitative study. BioMed Central Cardiovascular Disorders 17: 77.

2. Cavalheri V, Straker L, Gucciardi DF, Gardiner PA, Hill K 2016 Changing physical activity and sedentary behaviour in people with COPD. Respirology 21: 419-426.

3. Collins E, Langbein WE, Dilan-Koetje J, Bammert C, Hanson K, Reda D, Edwards L 2004 Effects of exercise training on aerobic capacity and quality of life in individuals with heart failure. Heart and Lung 33: 154-161.

4. Conraads VM, Deaton C, Piotrowicz E, Santaularia N, Tierney S, Piepoli MF, Pieske B, Schmid JP, Dickstein K, Ponikowski PP, et al. 2012 Adherence of heart failure patients to exercise: barriers and possible solutions: a position statement of the Study Group on Exercise Training in Heart Failure of the Heart Failure Association of the European Society of Cardiology. European Journal of Heart Failure 14: 451-458.

5. Cooper LB, Mentz RJ, Sun JL, Schulte PJ, Fleg JL, Cooper LS, Pina IL, Kraus WE, Whellan DJ, Keteyian S, et al. 2015 Psychosocial factors, exercise adherence, and outcomes in heart failure patients: Insights from Heart Failure: A Controlled Trial Investigating Outcomes of Exercise Training (HF-ACTION). Circulation Heart Failure 8: 1044-1051.

6. Davidson PM, Cockburn J, Newton PJ, Webster JK, Betihavas V, Howes, L, Owensby DO 2010 Can a heart failure-specific cardiac rehabilitation program decrease hospitalizations and improve outcomes in high-risk patients? European Journal of Cardiovascular Prevention and Rehabilitation 17: 393-402.

7. Deka P, Pozehl B, Williams MA, Yates B 2017 Adherence to recommended exercise guidelines in patients with heart failure. Heart Failure Reviews 22: 41-53. 
8. Doukky R, Mangla A, Ibrahim Z, Poulin MF, Avery E, Collado FM, Kaplan J, Richardson D, Powell LH 2016 Impact of physical inactivity on mortality in patients with heart failure. The American Journal of Cardiology 117: 1135-1143.

9. Eckel RH, Jakicic JM, Ard JD, de Jesus JM, Houston Miller N, Hubbard VS, Lee IM, Lichtenstein AH, Loria CM, Millen BE, et al. 20142013 AHA/ACC guideline on lifestyle management to reduce cardiovascular risk: a report of the American College of Cardiology/American Heart Association Task Force on Practice Guidelines. Journal of the American College of Cardiology 63: 2960-2984.

10. Evangelista LS, Cacciata M, Stromberg A, Dracup K 2017 Dose-response relationship between exercise intensity, mood states, and quality of life in patients with heart failure. The Journal of Cardiovascular Nursing 32: 530-537.

11. Furlanetto KC, Donaria L, Schneider LP, Lopes JR, Ribeiro M, Fernandes KB, Hermandes NA, Pitta F 2017 Sedentary behavior is an independent predictor of mortality in subjects with COPD. Respiratory Care 62: 579-587.

12. Haedtke C, Smith M, VanBuren J, Klein D, Turvey C 2017 The relationships among pain, depression, and physical activity in patients with heart failure. The Journal of Cardiovascular Nursing 32: E21-E25.

13. Hammill BG, Curtis LH, Schulman KA, Whellan DJ 2010 Relationship between cardiac rehabilitation and long-term risks of death and myocardial infarction among elderly Medicare beneficiaries. Circulation 121: 63-70.

14. Holland AE, Spruit MA, Troosters T, Puhan MA, Pepin V, Saey D, McCormack MC, Carlin BW, Sciurba FC, Pitta F, et al. 2014 An official European Respiratory Society/American Thoracic Society technical standard: field walking tests in chronic respiratory disease. The European Respiratory Journal 44: 1428-1446.

15. Houchen-Wolloff L, Watt A, Bryce S, Singh S 2016 Delivering early rehabilitation after an exacerbation of heart failure: is it feasible? A short report. The British Journal of Cardiology 23: doi:10.5837/bjc.2016.00. 
16. Keteyian SJ, Leifer ES, Houston-Miller N, Kraus WE, Brawner CA, O'Connor CM, Whellan DJ, Cooper LS, Fleg JL, Kitzman DW, et al. 2012. Relation between volume of exercise and clinical outcomes in patients with heart failure. Journal of the American College of Cardiology 60: 1899-1905.

17. Klompstra L, Jaarsma T, Stromberg A 2015 Physical activity in patients with heart failure: barriers and motivations with special focus on sex differences. Patient Preferance and Adherence 9: 1603-1610.

18. Liu MH, Wang CH, Tung TH, Kuo LT, Chiou AF 2018 Effects of a multidisciplinary disease management program with or without exercise training for heart failure patients: Secondary analysis of a randomized controlled trial. International Journal of Nursing Studies 87: 94-102.

19. Mandic S, Tymchak W, Kim D, Daub B, Quinney HA, Taylor D, Al-Kurtass S, Haykowsky MJ 2009 Effects of aerobic or aerobic and resistance training on cardiorespiratory and skeletal muscle function in heart failure: a randomized controlled pilot trial. Clinical Rehabilitation 23: 207-216.

20. Mesquita R, Meijer K, Pitta F, Azcuna H, Goertz YMJ, Essers JMN, Wouters EFM, Spruit MA 2017. Changes in physical activity and sedentary behaviour following pulmonary rehabilitation in patients with COPD. Respiratory Medicine 126:122-129.

21. Mudge AM, Denaro CP, Scott AC, Meyers D, Adsett JA, Mullins RW, Suna JM, Atherton JJ, Marwick TH, Scuffham P, et al. 2018 Addition of supervised exercise training to a post-hospital disease management program for patients recently hospitalized with acute heart failure. The EJECTION-HF Randomized Phase 4 Trial. JACC Heart Failure 6: 143-152.

22. Mudge AM, Denaro CP, Scott AC, Atherton JJ, Meyers DE, Marwick TH, Adsett JA, Mullins RW, Scuffham P, et al. 2011 Exercise training in recently hospitalized heart failure patients enrolled in a disease management program: design of the EJECTIONHF randomized controlled trial. European Journal of Heart Failure 13: 1370-1375. 
23. O'Connor CM, Whellan DJ, Lee KL, Keteyian SJ, Cooper LS, Ellis SJ, Leifer ES, Kraus WE, Kitzman DW, Blumenthal JA, et al. 2009 Efficacy and safety of exercise training in patients with chronic heart failure: HF-ACTION randomized controlled trial. JAMA. 301: 1439-1450.

24. Park LG, Schopfer DW, Zhang N, Shen H, Whooley MA 2017 Participation in Cardiac Rehabilitation Among Patients With Heart Failure. Journal of Cardiac Failure 23: 427431.

25. Piepoli MF, Binno S, Corra U, Seferovic P, Conraads V, Jaarsma T, Schmid JP, Filippatos G, Ponikowski PP 2015 ExtraHF survey: the first European survey on implementation of exercise training in heart failure patients. European Journal of Heart Failure 17: 631-638.

26. Sagar VA, Davies EJ, Briscoe S, Coats AJ, Dalal HM, Lough F, Rees K, Singh S, Taylor RS. 2015 Exercise-based rehabilitation for heart failure: systematic review and meta-analysis. Open Heart 2: e000163.

27. Sato N, Origuchi H, Yamamoto U, Takanaga Y, Mohri, M 2012 The importance of daily physical activity for improved exercise tolerance in heart failure patients with limited access to center-based cardiac rehabilitation. Experimental and Clinical Cardiology 17: 121-124.

28. Spruit MA, Pitta F, McAuley E, ZuWallack RL, Nici L 2015 Pulmonary rehabilitation and physical activity in patients with chronic obstructive pulmonary disease. American Journal of Respiratory and Critical Care Medicine 192: 924-933.

29. Sutherland N, Harrison A, Doherty P 2018 Factors influencing change in walking ability in patients with heart failure undergoing exercise-based cardiac rehabilitation. International Journal of Cardiology 268: 162-165. 
30. van der Wal MH, van Veldhuisen DJ, Veeger NJ, Rutten FH, Jaarsma T 2010 Compliance with non-pharmacological recommendations and outcome in heart failure patients. European Heart Journal 31: 1486-1493.

31. Vromen T, Kraal JJ, Kuiper J, Spee RF, Peek N, Kemps HM 2016 The influence of training characteristics on the effect of aerobic exercise training in patients with chronic heart failure: A meta-regression analysis. International Journal of Cardiology 208: 120127.

32. Waring T, Gross K, Soucier R, ZuWallack R 2017 Measured physical activity and 30day rehospitalization in heart failure patients. Journal of Cardiopulmonary Rehabilitation and Prevention 37: 124-129. 
Table 1. Participant characteristics

\begin{tabular}{|c|c|}
\hline Characteristics & $\begin{array}{c}\text { Participants } \\
n=140\end{array}$ \\
\hline Age, mean years (SD) & $62(15)$ \\
\hline Male gender, $\mathrm{n}(\%)$ & $108(77)$ \\
\hline $\mathrm{EF}<40 \%, \mathrm{n}(\%)$ & $108(77)$ \\
\hline New diagnosis HF, $\mathrm{n}(\%)$ & $82(58.6)$ \\
\hline NYHA, $\mathrm{n}(\%)$ & \\
\hline $\mathrm{I}$ & $25(18)$ \\
\hline II & $73(52)$ \\
\hline III & $26(19)$ \\
\hline IV & $16(11)$ \\
\hline HF aetiology, $\mathrm{n}(\%)$ & \\
\hline Ischaemic & $52(37)$ \\
\hline Hypertensive & $25(18)$ \\
\hline Idiopathic & $38(27)$ \\
\hline Other & $25(18)$ \\
\hline AF present, n (\%) & $53(38)$ \\
\hline Hypertension, n (\%) & $88(62.9)$ \\
\hline Diabetes, $\mathrm{n}(\%)$ & $48(34.3)$ \\
\hline
\end{tabular}




\begin{tabular}{|l|c|}
\hline COPD present, $\mathrm{n}(\%)$ & $34(24.3)$ \\
\hline Chronic renal impairment, $\mathrm{n}(\%)$ & $31(22.1)$ \\
\hline Anaemia present, (Hb $<120 \mathrm{~g} / \mathrm{L}$ males & $34(27)$ \\
and $<110 \mathrm{~g} / \mathrm{L}$ females), $\mathrm{n}(\%)$ & \\
\hline Depression present (GDS $>5), \mathrm{n}(\%)$ & $46(35)$ \\
\hline Multimorbidity CCI $\geq 3, \mathrm{n}(\%)$ & $66(47)$ \\
\hline Disability present (IADL $>1), \mathrm{n}(\%)$ & $73(52)$ \\
\hline Baseline 6MWD, mean metres (SD) & $361(127)$ \\
\hline Medications & $125(89.3)$ \\
ACEi/ ARB & $127(90.7)$ \\
Beta-blocker & $52(37.1)$ \\
MRA & $19(85.0)$ \\
\hline Digoxin & \\
\hline
\end{tabular}

ACEi, Angiotensin converting enzyme inhibitor; AF, Atrial fibrillation; ARB, Angiotensin receptor blocker; CCI, Charlson comorbidity index; COPD, Chronic obstructive lung disease; EF, Ejection fraction; GDS, Geriatric depression scale; Hb, Hemoglobin; IADL, Instrumental activities of daily living; MRA, Mineralocorticoid receptor antagonist; NYHA, New York Heart Association 
Table 2. Association between exercise variables and improvement in 6MWD

\begin{tabular}{|l|c|c|}
\hline & Improved 6MWD, n (\%) & p value \\
\hline Attendance frequency $\geq 12$ sessions & $34 / 52(65.9)$ & 0.003 \\
Attendance frequency $<12$ sessions & $23 / 62(37.1)$ & \\
\hline Attendance duration $\geq 6$ weeks & $53 / 101(52.5)$ & 0.141 \\
Attendance duration $<6$ weeks & $4 / 13(30.8)$ & \\
\hline PA baseline $\geq 150$ minutes & $44 / 88(50)$ & 0.347 \\
PA baseline $<150$ minutes & $53 / 122(43.4)$ & \\
\hline PA at 12 week follow-up $\geq 150$ minutes & $80 / 161(49.7)$ & \\
PA at 12 week follow-up $<150$ minutes & $16 / 48(33.3)$ & \\
& & \\
\hline
\end{tabular}

6MWD, 6 minute walk distance, PA; Physical activity 


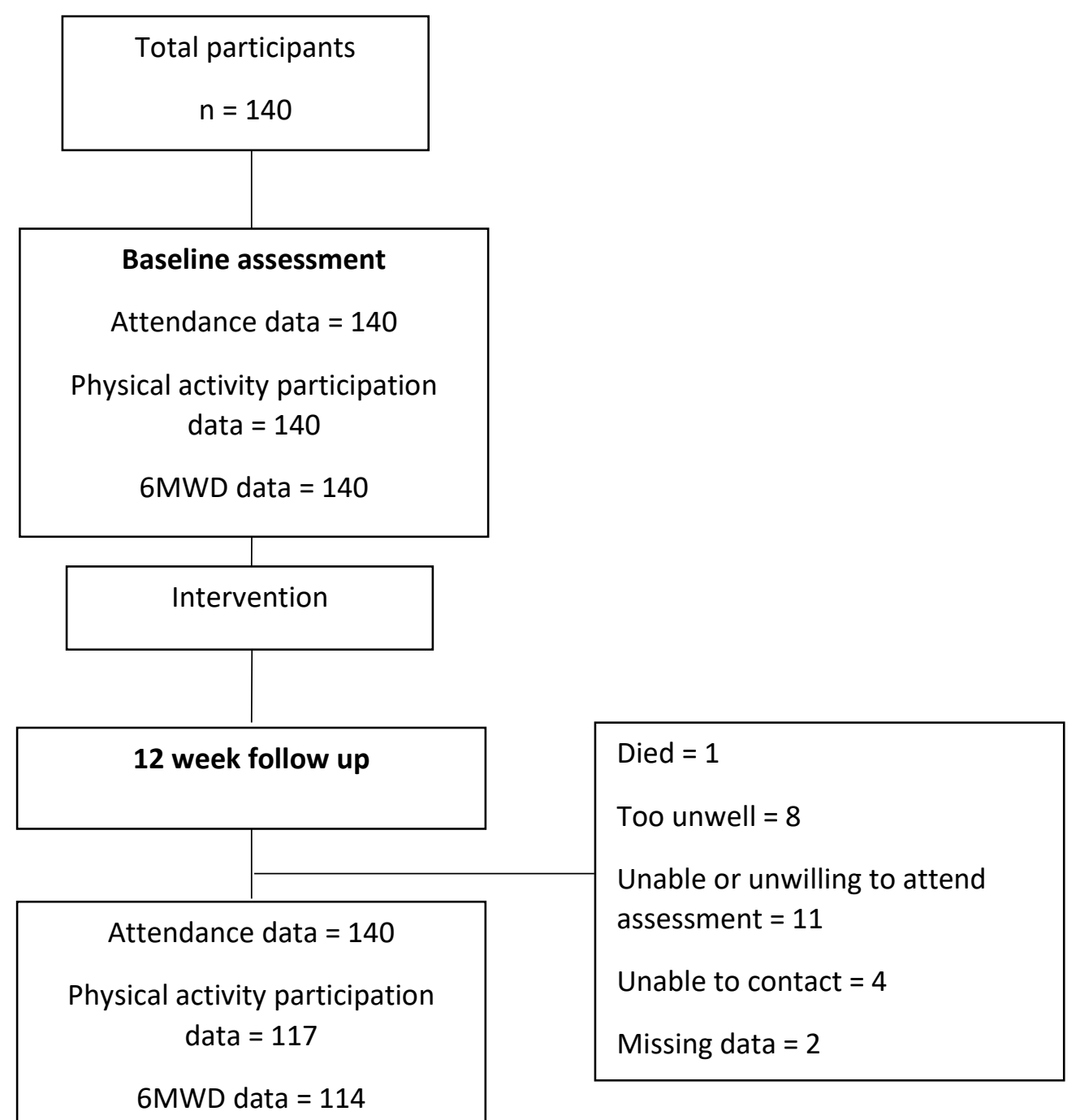

Figure 1. Flow diagram of participants and available data at baseline and $\mathbf{1 2}$ week follow up 6MWD, 6 minute walk distance 


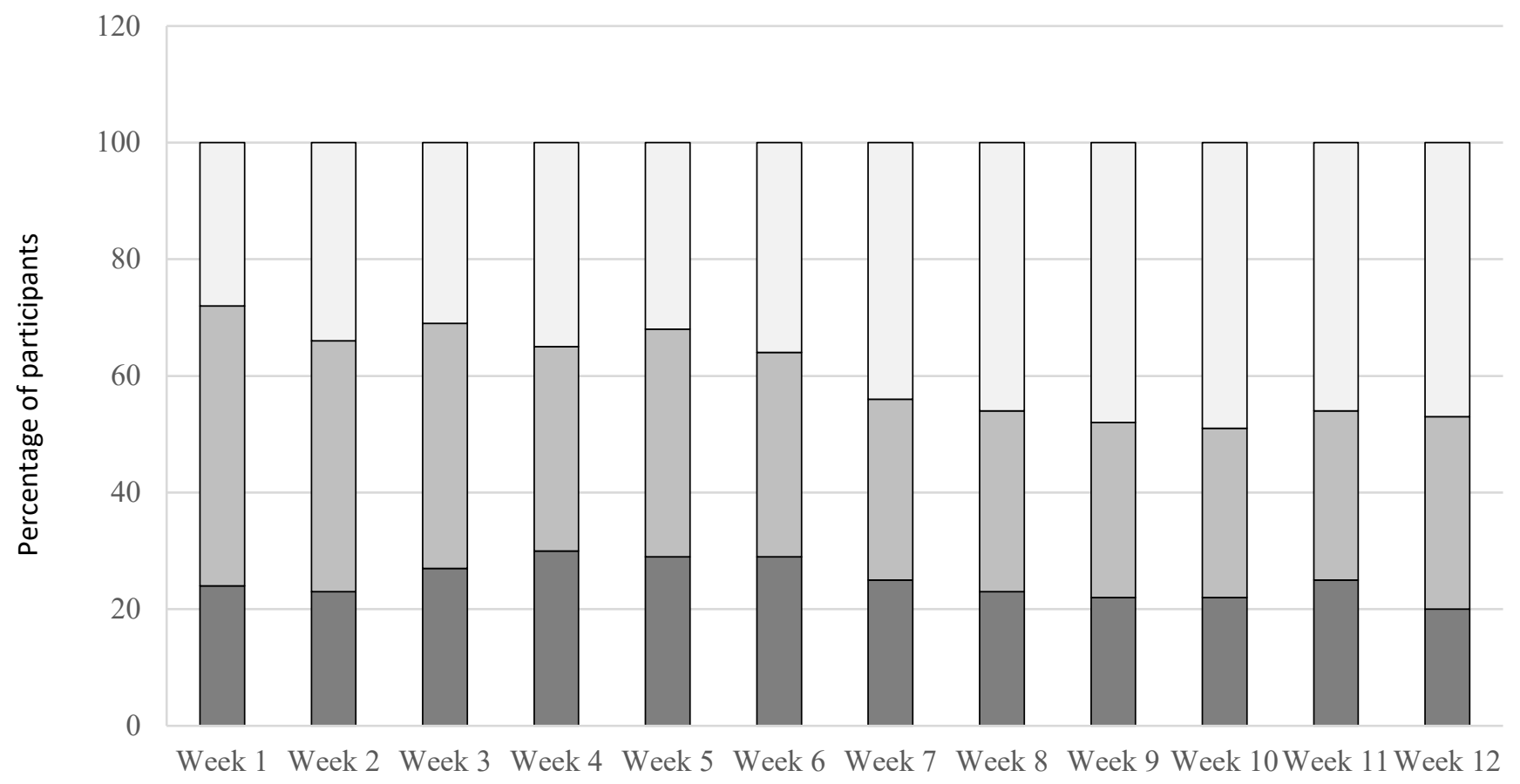

\author{
Week number \\ $\square$ Attended x2/wk $\square$ Attended x1/wk $\square$ Attended 0/wk
}

Figure 2. Percentage of participants who attended the exercise programme according to session frequency and week of attendance. 


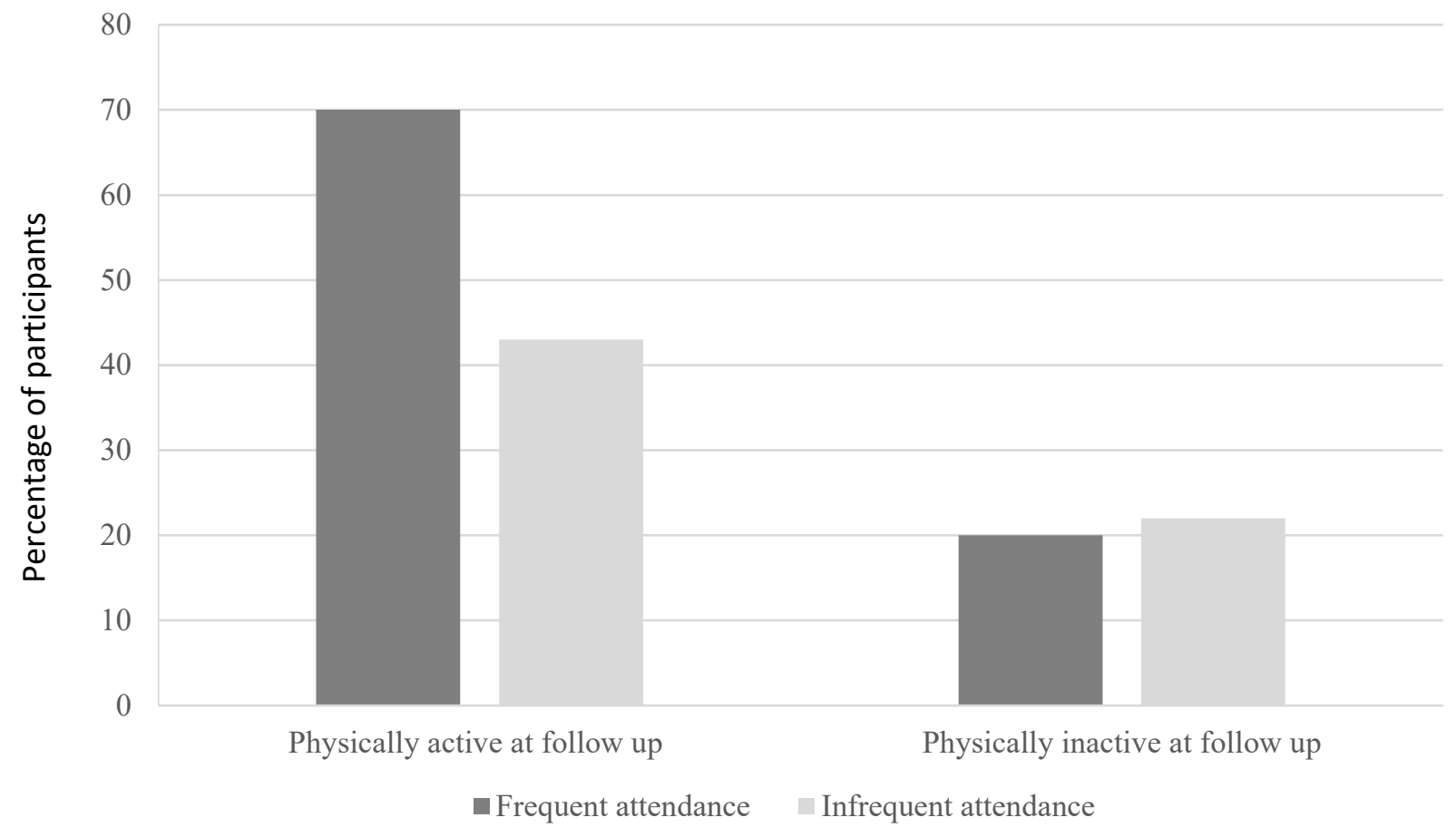

Figure 3. Percentage of participants who improved 6MWD at week 12 according to exercise programme attendance frequency and being physically active at 12 weeks 